\title{
Effect of Curcumin on Liver Damage Caused by Cisplatin in Rats
}

\author{
Büşra Şen Halıcıoğlu ${ }^{1 *}$, Hülya Birinci ${ }^{1}$, Mehmet Yüncü ${ }^{1}$ \\ ${ }^{1}$ Gaziantep University, Faculty of Medicine, Department of Histology \& Embryology, \\ Gaziantep, Turkey.
}

\begin{abstract}
The toxic effects that occur in many patients receiving chemotherapy seriously affect the liver. Cisplatin used in many types of cancer causes hepatotoxicity by increasing oxidative stress in the liver. Curcumin is a compound with antioxidant, antifibrogenic and anticarcinogenic features. Due to these healing effects of curcumin, it is recommended in many areas as an antioxidant. In this study, the effect of the antioxidant properties of curcumin on the liver of rats given cisplatin was investigated. For this purpose, 26 adult male Wistar Albino rats were divided into 4 groups. Control group rats were given DMSO for 6 days. Curcumin group rats were given intraperitoneally 200mg/kg curcumin for 6 days. In the Cisplatin + Curcumin group, $200 \mathrm{mg} / \mathrm{kg}$ curcumin intraperitoneally was given daily for 6 days and a single dose of $20 \mathrm{mg} / \mathrm{kg}$ cisplatin intraperitoneally was given on the 3rd day only. On the 7th day, after the animals were sacrificed, liver tissue was taken for light and electron microscopy analysis. As a result, it showed that cisplatin causes steatosis in hepatocytes, congestion in sinusoids and portal areas even when administered acutely. Cisplatin is thought to cause these effects by increasing oxidative stress in the liver. Curcumin was observed to be alleviating hepatotoxic effects cisplatin which was revealed by both light and electron microscopic findings.
\end{abstract}

Key words: Cisplatin, Curcumin, Hepatotoxicity

\section{Introduction}

Cisplatin is an antitumor agent containing heavy metal platinum. It specifically inhibits cell proliferation and also has antibacterial properties $(1,2)$. It is used in various cancer types such as ovarian, testicular, head and neck cancers $(3,4)$. It is known that in the molecular mechanism of cisplatin, it binds to the purine bases in the DNA of cancerous cells and causes DNA breaks and stop dividing of these cells and eliminates them. Cisplatin reaches its highest level in plasma within one hour after intravenous administration. It can reach high concentrations in the liver, kidneys, and intestines. Although the minimum lethal dose of cisplatin is unknown, taking 180-480 $\mathrm{mg} / \mathrm{ml}$ intravenously, although not lethal, may cause significant toxicity (5). The most important side effects of cisplatin are hepatotoxicity and nephrotoxicity (3).

* Corresponding author: Büşra Şen Halıcıoğlu, Medical Faculty of Gaziantep University, Department of Histology \& Embryology, E-mail: busrasen89@ gmail.com, ORCID ID: 0000-0003-4089-5243. 
Even the treatment dose used as tumor suppressor can cause hepatotoxicity (6). Although the damage inducing mechanism of cisplatin is not well known, some evidence suggests that cisplatin induces hepatoxicity by oxidative stress (7).

Curcumin, which is generally used as a food color or spice, is grown in many Asian countries, especially India and China. Curcumin, known as turmeric in Turkey are natural organic substance derived from the plant Curcuma longa (8, 9). Curcumin is a hydrophobic substance containing two phenolic rings. Curcumin cannot be dissolved in water and ether, but soluble in ethanol, chloroform, dichloromethane, acetone and DMSO (10). Studies on curcumin have reported that curcumin has antioxidant, anticancer, antiinflammatory and antiseptic properties (11, 12). Different studies have shown that curcumin reduces the level of cholesterol in the blood, prevents HIV replication, suppresses the symptoms of diseases such as Type2 diabetes, rheumatoid arthritis, multiple sclerosis and Alzheimer's, accelerates wound healing, increases bile secretion and prevents liver damage (8). The liver is a vital organ for the body and is responsible for maintaining metabolic functions. Liver diseases are one of the major diseases seen in worldwide. Many factors such as certain chemotherapeutic agents, xenobiotics, immunosuppressants, heavy metals and alcohol can cause liver damage (13). Cisplatin, which is used in the treatment of various cancer types, is a chemotherapy drug with many side effects. One of the important side effects is hepatotoxicity. It causes oxidative stress to increase by increasing free radicals, especially in the liver (7). In this study, liver damage caused by cisplatin, a chemotherapeutic agent, was demonstrated. At the same time, when curcumin was given to rats exposed to cisplatin, the changes in their livers were demonstrated by light and electron microscopy.

\section{Materials and methods}

\section{Ethical approval}

All procedures performed during the experiment were approved by the Animal Experiments Local Ethics Committee of Kahramanmaraş Sütçü İmam University with the ethics committee decision dated 21.10.2014.

\section{Animals}

A total of 26 adult male Wistar Albino rats weighing between 200- 250 grams were used in the study. In Kahramanmaraş Şütçü Imam University Experimental Animals Laboratory, where the animals were kept in 12 hours light and 12 hours dark environment, proper nutrition and free drinking were provided in suitable cages. Animals were divided into 4 groups as control group, cisplatin group, curcumin group and cisplatin + curcumin group. In control groups $(\mathrm{n}=5)$ DMSO $2 \mathrm{cc} / \mathrm{kg}$ was given intraperitoneally (i.p) for 6 days. In Curcumin group $(n=7)$, curcumin $200 \mathrm{mg} / \mathrm{kg}$ was given intraperitoneally (i.p.) as a single daily dose for 6 days. Cisplatin group $(n=7)$ was given a single dose of cisplatin $20 \mathrm{mg} / \mathrm{kg}$ i.p. on the $3 \mathrm{rd}$ day of the experiment. Curcumin + Cisplatin Group received a single dose of curcumin $200 \mathrm{mg} / \mathrm{kg}$ i.p. for 6 days and a single dose of cisplatin $20 \mathrm{mg} / \mathrm{kg}$ i.p. on the $3 \mathrm{rd}$ day. On the 7 th day of the experiment, all rats were sacrificed.

\section{Preparation of Tissues for Light}

\section{Microscopy}

Liver tissues taken after dissection were fixed in $10 \%$ formaldehyde. The fixed tissues were passed through increasing 
alcohol series after standing in running water for overnight. Tissues held in xylene for transparency were embedded in paraffin blocks. Then, sections of $5 \mu \mathrm{m}$ thickness were taken from these blocks and hematoxylin-eosin staining was performed. The stained sections were examined with a light microscope (Leica DM 750) and photographed with camera (Olympus DP71).

Preparation of Tissues for Electron Microscopy

The liver tissues were cut in $1 \mathrm{~mm} 3$ volumes and immediately taken into $5 \%$ glutaraldehyde solution for primary fixation. Tissues were kept in osmium tetroxide for 2 hours for secondary fixation. The tissues, which were then washed with buffer and passed through the increased alcohol series, were kept in propylene oxide and resin. Finally, the tissues were placed in suitable embedding capsules after overnight in resin and fresh resin was poured over the tissues. Thus, the tissues were made able to be sectioned in the ultramicrotome. $10-20 \mathrm{~nm}$ thick sections were taken from the tissues placed in the ultramicrotome. The sections were placed on copper grids and stained with uranyl acetate. The prepared tissues were examined under the electron microscope and images were photographed.

\section{Results}

\section{Light Microscopic Evaluations}

In light microscopic images of the control group liver tissue, organized hepatocyte cords and a normal liver tissue were observed. Portal areas around the vena centralis were seen properly. It was observed that the sinusoids were of normal width (Figure 1A). Similar findings were found in the liver tissue of the curcumin group as well as the control group. There were regular hepatocyte cords and normal sinusoids around the portal areas (Figure 1B). A large amount of micro vesicular steatosis was observed in the liver tissue of the cisplatin group, especially in hepatocytes around the portal area. Enlargement and congestion in the sinusoids and congestion in the central vein were observed (Figure 1C). Similar findings were found in the Cisplatin + Curcumin group as well as in the control group. Steatosis was seen within hepatocytes, with only a small amount around the central vein (Figure 1D).

\section{Electron Microscopic Evaluations}

In the electron microscopic images of the control group, hepatocytes were observed with a normal nucleus in the center and a polygonal structure with well distinct cell borders. Numerous endoplasmic reticulum and mitochondria were found in hepatocytes, which seem to be rich in organelles. Small amounts of lysosomes were observed in some areas. Bile canaliculi were observed normally among the hepatocytes. Erythrocytes and plasma cells were seen in the sinusoid (Figure 2A). Electron-microscopic images of the curcumin group showed areas similar to the control group. Regular round nuclei and properly structured GERs and mitochondria were observed in the center. Sinusoid walls were seen together with flat sinusoid endothelial cells in the sections. Occasionally, lysosomes were found in the cytoplasm (Figure 2B). In cisplatin group electron microscope images, hepatocyte nuclei were generally irregularly shaped. 

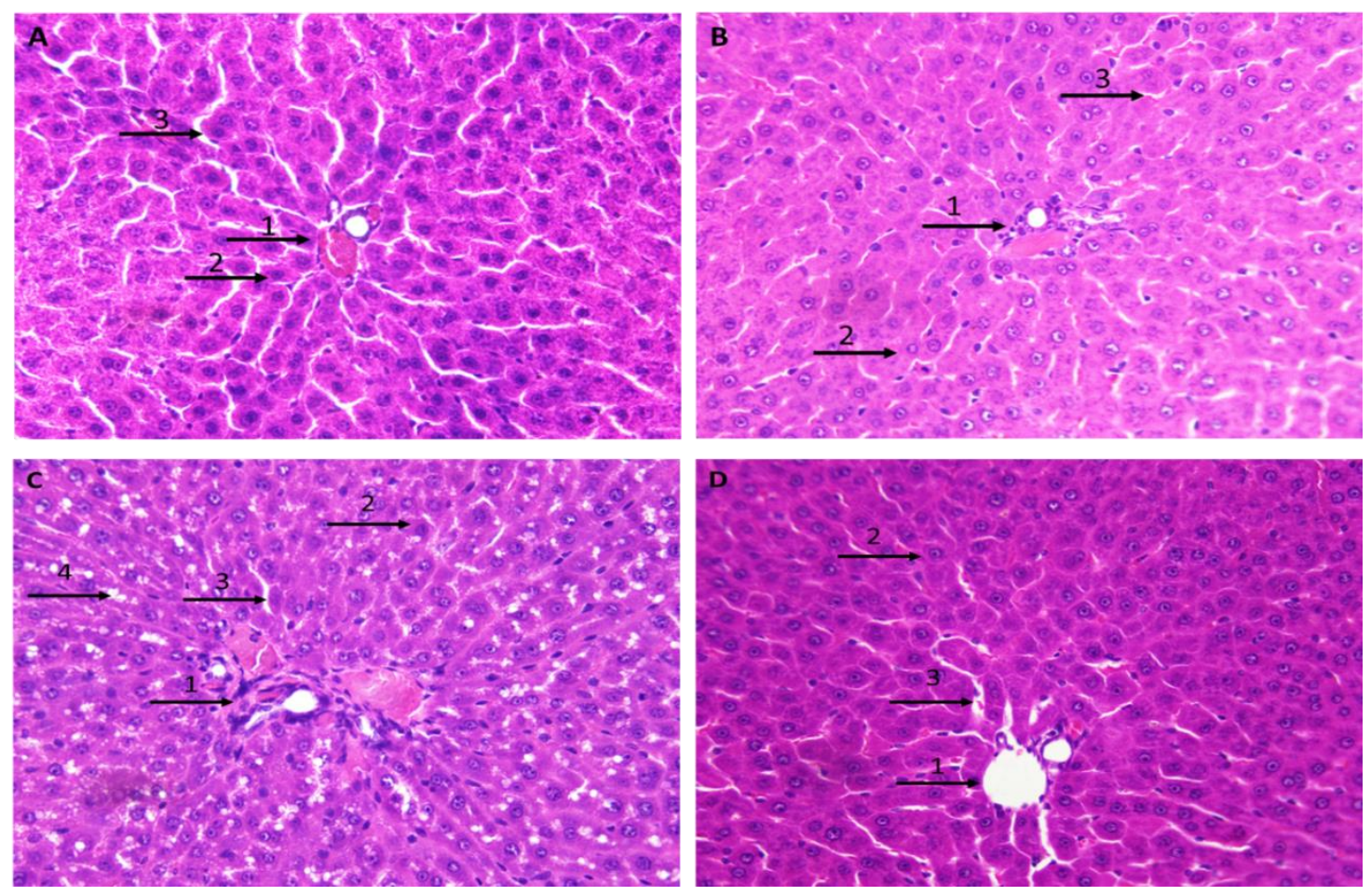

Figure 1: Liver tissue H\&E staining. Scala bar:10 $\mu$ m. A. Control group B. Curcumin group C. Cisplatin group D. Curcumin + Cisplatin group. 1. Portal area, 2. Hepatocytes, 3. Sinusoids, 4. Steatosis.
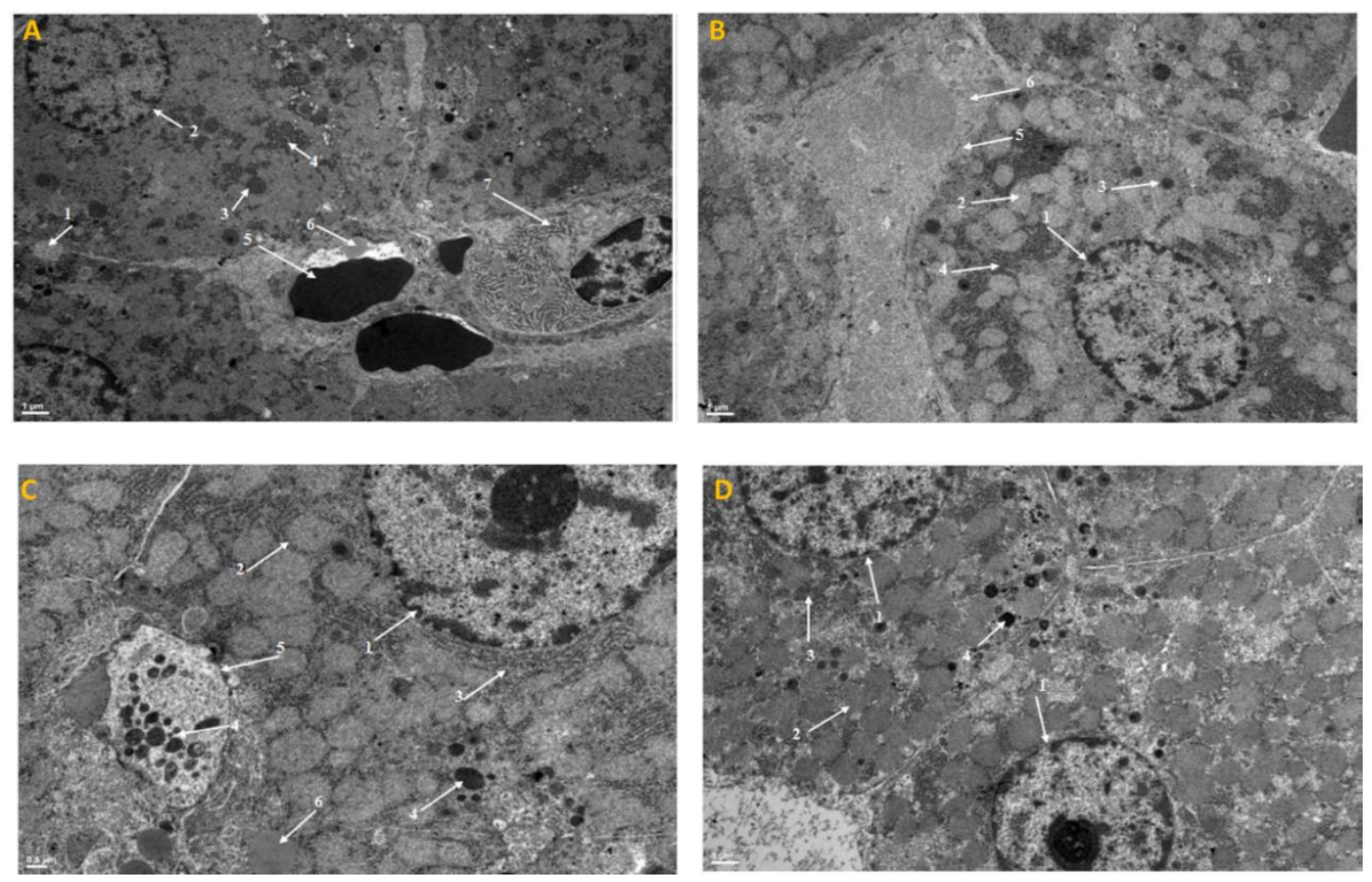

Figure 2: Liver tissue, electron microscopic photograph. A. Control group; 1) Bile canaliculus, 2) Hepatocyte nucleus, 3) Lysosome, 4) Granular endoplasmic reticulum, 5) Erythrocyte, 7) Plasma cell B. Curcumin group; 1) Hepatocyte nucleus, 2) Mitochondria, 3) Lysosome, 4) Granular endoplasmic reticulum, 5) Sinusoid endothelium, 6) Sinusoid C. Cisplatin group; 1) Hepatocyte nucleus, 2) Mitochondria, 3) Granular endoplasmic reticulum, 4) Lysosome, 5) Kupffer Cell, 6) Lipid Droplets D. Curcumin + Cisplatin group; 1) Hepatocyte nucleus, 2) Mitochondria, 3) Granular endoplasmic reticulum, 4) Lysosome. 
While the granular endoplasmic reticulum was more in the control groups, it was observed to be less in the cisplatin group. Swelling and vacuolization were observed in mitochondria. Kupffer cells rich in lysosomes were observed. Increased lipid droplets observed (Figure 2C). Electron microscopy images of the curcumin + cisplatin group showed similar findings to the control and curcumin groups. Usually, smooth round nuclei, GER and mitochondria were seen in the center. Glycogen particles and a small amount of lipid droplets were observed. Increased lysosomes were seen compared to the control and only curcumin group (Figure 2D).

\section{Discussion}

The liver is exposed to many toxic substances such as chemotherapeutic agents, heavy metals and alcohol due to its important physiological functions such as maintenance of metabolic homeostasis and detoxification of harmful metabolites. . Therefore, it is a clinical condition with liver toxicity all over the world $(14,15)$. Oxidative stress plays an important role in the emergence of liver diseases. It causes many liver diseases such as cirrhosis, especially by initiating fibrosis in the liver. Antioxidants have an important role in preventing liver fibrosis caused by oxidative stress and diseases that will develop as a result of fibrosis. Chemotherapy drugs have been used for cancer treatment for many years. Especially high doses of cisplatin cause hepatotoxicity. Since there is not enough information about how cisplatin causes hepatotoxicity, the mechanism of inducing toxic effect is not known much. However, recent studies suggest that cisplatin causes hepatotoxicity by triggering oxidative stress $(6,16)$. Oxidative stress caused by the increase of free radicals in the cell or the reduction of antioxidant structures triggers the formation of fibrogenesis in the liver by increasing cytokines such as transforming growth factor- $\beta$ (TGF- $\beta$ ), interlukin-6 (IL-6) and tumor necrosis factor- $\alpha(\mathrm{TNF} \alpha)(15,17)$. Cisplatin also causes structural damage to mitochondria, decreasing membrane fluidity and increasing mitochondrial membrane stiffness (18). In addition, oxidation of cardiolipin in mitochondria induces apoptotic pathway in cells by increasing cytochrome c and caspase activation (19). In a study by S.Palipoch et al., Liver congestion, disorganization of hepatocyte cords and tissue damage were observed in rats exposed to cisplatin (20). In the histopathological findings obtained as a result of our study, congestion was observed in the portal areas, vena centralis and sinusoids in the cisplatin group. A large amount of micro vesicular steatosis was observed in hepatocytes around the portal areas. Also increased Kupffer cells were shown. These histopathological findings support the literature. Curcumin used in our study is a plant that has been studied extensively due to its antioxidant, anti-inflammatory, antimicrobial and anticancer properties. Despite this, the molecular mechanism of action has not been fully resolved (21, 22). However, studies show that the antioxidant mechanism of curcumin occurs by inhibiting oxidative enzymes or by affecting oxidative pathways (23). Curcumin is known to be nontoxic even if taken in high doses. However, long-term use of high doses in some mice and rat species may cause hepatotoxicity (24). Many in vitro studies show that curcumin has a protective and healing effect against 
chronic liver diseases. In in vivo studies in animals, it has been also said that it has antifibrogenic properties by inactivating some pathways in liver damage $(22,25)$. In our study, a significant decrease was observed in hepatocyte steatosis in the group given curcumin with cisplatin compared to the group given only cisplatin. There was a significant decrease in congestions occurring in the central vein and portal area. Although congestion was observed in sinusoids in some areas, it was observed that congestion decreased compared to the group that was given only cisplatin. Similar to our study, Ying Wang et al. showed the liver damage caused by cisplatin. The liver structure was severely affected in the cisplatin group, as in our study. It has been stated that most of the nuclei have a picnotic appearance, their size is reduced, and an uneven distribution of chromatin is observed. The cytoplasm of many hepatocytes had a foamy appearance due to dense vacuoles. In the curcumin + cisplatin group, they observed less abnormal nucleolus and less cytoplasmic vacuolization (26).

\section{Conclusion}

In this study, it was shown that cisplatin exposure affects parenchymal and nonparenchymal cells in liver tissue and curcumin can prevent these effects due to its antioxidant properties. The findings we obtained in our study also supports the information in the literature. In addition to our work in order to fully enlighten the subject, further studies are needed to reveal the molecular effects of curcumin on different organs and metabolic pathways in order to better understand the effects of curcumin on the human body.

\section{Conflict of interests}

The authors declare no conflict of interests.

\section{Acknowledgement}

All researches contributed equally to the study. This study was supported by Gaziantep University Scientific Research Projects Unit with project number of TF.15.10.

\section{References}

1. Chirino YI, Pedraza-Chaverri J. Role of oxidative and nitrosative stress in cisplatin-induced nephrotoxicity. Experimental and Toxicologic Pathology. 2009;61(3):223-42.

2. Murray D, Mirzayans R. Cellular Responses to Platinum-Based Anticancer Drugs and UVC: Role of p53 and Implications for Cancer Therapy. Int J Mol Sci. 2020;21(16).

3. Zheng X-N, Wang X-W, Li L-Y, et al. Puerh tea powder preventive effects on cisplatininduced liver oxidative damage in Wistar rats. Asian Pac J Cancer Prev. 2014;15(17):7389-94.

4. $\mathrm{Yu} \mathrm{D,} \mathrm{Gu} \mathrm{J,} \mathrm{Chen} \mathrm{Y,} \mathrm{et} \mathrm{al.} \mathrm{Current}$ Strategies to Combat Cisplatin-Induced Ototoxicity. Frontiers in pharmacology. 2020;11:999.

5. Tsang RY, Al-Fayea T, Au H-J. Cisplatin overdose. Drug safety. 2009;32(12):1109-22.

6. Naqshbandi A, Khan W, Rizwan S, et al. Studies on the protective effect of flaxseed oil on cisplatin-induced hepatotoxicity. Human \& experimental toxicology. 2012;31(4):364-75.

7. Liao Y, Lu X, Lu C, et al. Selection of agents for prevention of cisplatin-induced hepatotoxicity. Pharmacological Research. 2008;57(2):125-31.

8. Aggarwal BB, Kumar A, Bharti AC. Anticancer potential of curcumin: preclinical and clinical studies. Anticancer research. 2003;23(1/A):363-98.

9. Sharifi-Rad J, Rayess YE, Rizk AA, et al. Turmeric and Its Major Compound Curcumin on Health: Bioactive Effects and Safety Profiles for Food, Pharmaceutical, Biotechnological and Medicinal Applications. Frontiers in pharmacology. 2020;11:01021.

10. Pandey A, Gupta R, Srivastava R. Curcumin-the yellow magic. Asian J Appl Sci. 2011;4(4):343-54.

11. Rivera-Espinoza $\mathrm{Y}$, Muriel $\mathrm{P}$. Pharmacological actions of curcumin in liver diseases or damage. Liver International. 2009;29(10):1457-66.

12. Farzaei MH, Zobeiri M, Parvizi F, et al. Curcumin in Liver Diseases: A Systematic Review 
of the Cellular Mechanisms of Oxidative Stress and Clinical Perspective. Nutrients. 2018;10(7).

13. Ingawale DK, Mandlik SK, Naik SR. Models of hepatotoxicity and the underlying cellular, biochemical and immunological mechanism (s): a critical discussion. Environmental toxicology and pharmacology. 2014;37(1):118-33.

14. Auger C, Alhasawi A, Contavadoo M, et al. Dysfunctional mitochondrial bioenergetics and the pathogenesis of hepatic disorders. Frontiers in cell and developmental biology. 2015;3:40.

15. Casas-Grajales S, Muriel P. Antioxidants in liver health. World journal of gastrointestinal pharmacology and therapeutics. 2015;6(3):59.

16. Waseem M, Bhardwaj M, Tabassum H, et al. Cisplatin hepatotoxicity mediated by mitochondrial stress. Drug and chemical toxicology. 2015;38(4):452-9.

17. Muriel P. Cytokines in liver diseases. Wiley Online Library; 2007.

18. Martins N, Santos N, Curti C, et al. Cisplatin induces mitochondrial oxidative stress with resultant energetic metabolism impairment, membrane rigidification and apoptosis in rat liver. Journal of Applied Toxicology: An International Journal. 2008;28(3):337-44.

19. Grattagliano I, Portincasa P, Palmieri VO, et al. Overview on the mechanisms of drug-induced liver cell death. Annals of Hepatology. 2002;1(4):162-8.
20. Palipoch S, Punsawad C, Koomhin P, et al. Hepatoprotective effect of curcumin and alphatocopherol against cisplatin-induced oxidative stress. BMC complementary and alternative medicine. 2014;14(1):111.

21. Gupta SC, Patchva S, Koh W, et al. Discovery of curcumin, a component of golden spice, and its miraculous biological activities. Clinical and experimental pharmacology and physiology. 2012;39(3):283-99.

22. Vera-Ramirez L, Pérez-Lopez P, VarelaLopez A, et al. Curcumin and liver disease. Biofactors. 2013;39(1):88-100.

23. Ak T, Gülçin İ. Antioxidant and radical scavenging properties of curcumin. Chemicobiological interactions. 2008;174(1):27-37.

24. Chainani-Wu N. Safety and antiinflammatory activity of curcumin: a component of tumeric (Curcuma longa). The Journal of Alternative \& Complementary Medicine. 2003;9(1):161-8.

25. Ghosh N, Ghosh R, Mandal V, et al. Recent advances in herbal medicine for treatment of liver diseases. Pharmaceutical biology. 2011;49(9):970-88.

26. Wang Y, Hu P-C, Gao F-F, et al. The protective effect of curcumin on hepatotoxicity and ultrastructural damage induced by cisplatin. Ultrastructural pathology. 2014;38(5):358-62. 